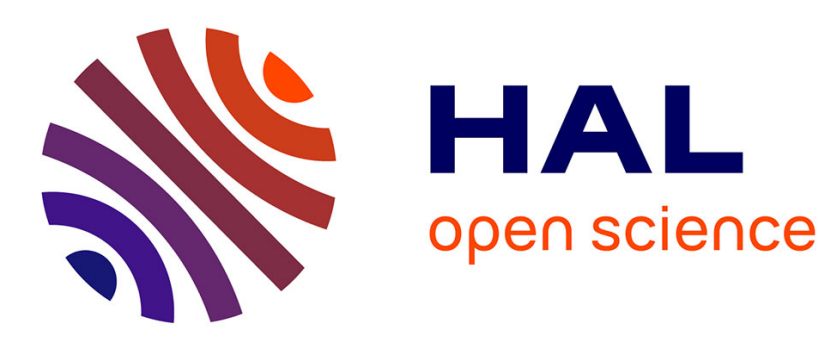

\title{
User Study: A Detailed View on the Effectiveness and Design of Tactile Charts
}

\author{
Christin Engel, Gerhard Weber
}

\section{To cite this version:}

Christin Engel, Gerhard Weber. User Study: A Detailed View on the Effectiveness and Design of Tactile Charts. 17th IFIP Conference on Human-Computer Interaction (INTERACT), Sep 2019, Paphos, Cyprus. pp.63-82, 10.1007/978-3-030-29381-9_5 . hal-02544546

\section{HAL Id: hal-02544546 \\ https://hal.inria.fr/hal-02544546}

Submitted on 16 Apr 2020

HAL is a multi-disciplinary open access archive for the deposit and dissemination of scientific research documents, whether they are published or not. The documents may come from teaching and research institutions in France or abroad, or from public or private research centers.
L'archive ouverte pluridisciplinaire HAL, est destinée au dépôt et à la diffusion de documents scientifiques de niveau recherche, publiés ou non, émanant des établissements d'enseignement et de recherche français ou étrangers, des laboratoires publics ou privés. 


\title{
User Study: A Detailed View on the Effectiveness and Design of Tactile Charts
}

\author{
Christin Engel and Gerhard Weber \\ TU Dresden, Faculty of Computer Science, Chair of Human-Computer-Interaction, \\ 01062 Dresden \\ \{christin.engel, gerhard.weber\}@tu-dresden.de
}

\begin{abstract}
Charts such as bar or pie charts are often used to represent data and their relation. Tactile charts are widely used to enable blind and visually-impaired people to explore charts through the sense of touch. Effective tactile chart design differs from its visual counterpart due to sequential nature of touch. Accordingly, in a study with 48 blind and visually-impaired participants we investigated the preferences for chart types, design features and errors in reading data values. We developed bar, line and pie charts as well as scatterplots with different layouts and novel design properties. Participants answered questions concerning the readability, content and data, specific design aspects as well as a personal rating. Overall, participants answered $80 \%$ of nominal questions regarding minima, maxima, and comparisons, correctly. Blind participants achieved a corrected mean error rate of $4.5 \%$, when reading single points or intersections, for example. More specifically, we directly compare chart types, and discuss the results for specific design considerations (e.g. distances between bars, width of bars, design and use of grid lines in scatterplots) by comparing different charts.
\end{abstract}

Keywords: User Studies · Blind and Visually Impaired People · Tactile Chart Design

\section{Introduction}

Graphics are important to communicate and represent ideas, relations or spatial information. Information graphics such as bar or line charts are often used in our daily life to represent data and its meaning. Analysing data by using information visualisations is a common way to gain insights from the data. Information visualisations have been developed for the visual sense and make use of domain specific skills. Due to the preattentive perception, it is easy to quickly detect outliers or patterns in visualisations. The development of new information visualisation techniques is ongoing since Cleveland has presented his seminal work (e.g. [?]). The design and type of visualisation depends on the underlying data as well as the analysis tasks. Different visualisation types are suitable for specific analysis tasks such as details-on-demand, overview or filter [?]. Analysing data effectively is a main requirement in many professions and daily life. Blind and 
visually-impaired people cannot access information graphics and its underlying data. Therefore, accessibility guidelines such as BITV (Barrier-Free Information Technology Ordinance) state to provide a verbal description of non-textual content. The basic verbalisation of common chart types like bar charts, line charts or scatterplots is well defined. Existing guidelines (e.g. BANA guidelines [?]) recommend describing the elements and structure of a chart while providing the underlying data within a data table. This is not useful for complex charts or for understanding the content of the chart. Analysis of raw data in a table data is very difficult, whereby especially gaining an overview or detecting trends.

Tactile charts are suitable to provide access to charts for blind people. They comprise raised elements and can be perceived by the sense of touch two-handed. Colours in visual charts are replaced by line styles, symbols and textures to distinguish data sets from each other. Tactile charts allow blind and visuallyimpaired people to explore and analyse the represented data independently. In addition, tactile charts provide spatial information, which supports the understanding and memorability of the content.

Readability of tactile charts is influenced by their design. Most guidelines concerning tactile chart design recommend a design similar to the visual counterpart (e.g. [?,?,?]). Visual design differs from tactile design because the two senses have different perception properties. Given that tactile perception has a small resolution, tactile charts have to be adapted for the tactile sense [?,?]. In addition, there is not always a visual counterpart. Furthermore, existing guidelines are lacking of specific use cases of tactile charts. For example, BANA guidelines recommend avoiding grid lines in scatterplots because the purpose of this chart type is to show correlations. This may be true for some but not all use cases. Most guidelines are too general and do not provide precise design recommendations for effective tactile chart design. In addition, specifications of necessary distances or sizes of chart-specific elements are often only vaguely provided. For instance, the size of point symbols in scatterplots is determined by the required space between point symbols [?]. Other guidelines simply provide examples for transcriptions [?]. Prescher et al. [?] developed general guidelines for tactile graphics and provide clear examples and precise specifications. In the following, we investigate such general recommendations and their application to different chart types.

\section{Research on Tactile Chart Design}

There is initial research concerning tactile chart design. Panëels et al. [?] reviewed the field of haptic data visualisations for blind people, discussing haptic line, bar and pie charts regarding the representation, quantitative information, overview, exploration and automation. The authors show the wide range of multimodal approaches and force-feedback systems for the representation of haptic charts. They underline the need for effective visualisation methods. A few studies have highlighted the effectiveness of tactile charts. Watanabe et al. [?] compared the usability of a Braille table, a digital data table and a scatterplot with three 
blind students. Tactile scatterplot outperformed the other representation methods when identifying trends quickly. The authors repeated this study with ten blind participants aged from 19 to 27 years [?]. Second, Watanabe et al. used identical data sets to compare a tactile table, a tactile scatterplot and a digital table to ascertain which representation method is most effective. Their charts represent four relation types: linear, quadratic, inverse proportional and noncorrelated (12 data sets overall). They show blind people are able to identify relations in a scatterplot more quickly than in a tactile table or a digital table. Tactile chart was understood in the shortest time. At the same time, error rates between the three conditions were comparable.

Especially the design of grid lines in tactile charts is commonly discussed. In a study with twelve female students (six blind), Aldrich et al. [?] compared three formats for the representation of tactile line charts. They used a line chart with standard $\mathrm{x}$ and $\mathrm{y}$ axes without grid lines (L/no-grid), one with duplicated axes (box/no-grid) and one with duplicated axes and grid lines (box/grid). The box/grid condition led to the most accurate answers. The L/no-grid format achieved the shortest response time. Lederman et al. [?] evaluated four grid formats with 20 adult participants: no-grid, grid-on-graph (extended tick marks into graph area), grid-overlay (separate sheet served as an overlay) and grid-underlay where a separate sheet was placed underneath the chart. The study shows no correlation between age of blindness and the graph-reading performance of participants. The grid-on-graph and grid-underlay condition performed best. Barth et al. [?] evaluated line charts with an incised grid/raised data curve condition (grids were embossed on the other side of the sheet) with 24 blind students. This incised grid condition significantly improves the reading time in comparison with the raised-grid and no-grid condition. Their aimed to find effects of different design characteristics on graph-reading performance (extreme and intersections points, line tracing, reading time).

Goncu et al. [?] investigated several formats of bar charts with twelve blind participants. They created three formats for three types of bar charts (simple, grouped, stacked). Grid and values at the top of each bar led to the lowest error rate in comparison with the no grid/no values and grid/no values conditions. In addition, grid and values were preferred by participants. Surprisingly, the tactile data table was preferred over the tactile chart ( 6 participants, 8 data points).

More general research focuses on the discriminability of embossed elements. Culbert et al. [?] identified a set of eleven textures that are discriminable. Nolan et al. [?] defined a high legible set of eleven point symbols and seven line symbols designed for tactile maps. Furthermore, Prescher et al. [?] present a consistency set of nine tactile patterns that are recognisable over different production media.

Overall, research in tactile chart design only focuses on a few chart types, whereby line charts and bar charts are the most popular. There is initial research concerning the effectiveness of tactile charts. While some studies focus on the comparison with other data representation methods, others describe the effectiveness based on the reading performance. Effectiveness among different chart types has not been considered. Further research is necessary to understand their 
limitations [?]. Research should focus in particular on designing tactile charts for data analysis. Furthermore, design parameters are not evaluated for different chart types. For example, the use of grid lines is commonly discussed for line charts but not for scatterplots. The results of the studies are only comparable to a limited extent due to different design parameters. Current research indicates certain chart elements influence the readability of the chart. Many design features for specific chart types remains unclear (e.g. distance between bars, textures in bar charts, point symbols in scatterplots). Most studies only compare single data sets or data sets with few values and do not use real data sets (apart from Goncu et al. [?]). Accordingly, current studies do not evaluate real-world scenarios where the user has to understand the data and its meaning in the context to the given chart. Data sets often include only a few data points and rarely multiple data sets. The data is optimised for tactile output. For example, overlapping symbols in scatterplots, charts without clear correlations and outliers, thin columns, very close data lines or different point symbols in scatterplots had not yet been investigated. Several analysis tasks need to be explored such as identifying clusters in scatterplots. Furthermore, it is not known if certain design features are intuitively understood and whether blind people can access tactile charts independently. If tactile charts need to be designed differently for different user groups is unclear.

In this context, it is important to evaluate tactile charts with blind participants having a different background. This is a major problem with prior studies, because they were carried out with a small group of participants who are mostly homogeneous regarding their experiences or age (e.g. female students largely of a similar age [?]). Overall, there is a need to investigate the effectiveness and design of tactile charts based on different criteria through user studies. It remains unclear whether tactile charts can be understood by blind people who are unfamiliar with them.

Accordingly, we investigated several studies about tactile chart design. First, we analysed the design of tactile charts collected from online libraries, publications and transcribing institutes [?]. In an online survey with 71 sighted, visuallyimpaired and blind creators and users of tactile charts [?] we included questions about the current production process of tactile charts, chart types known, exploration strategies as well as the design of tactile charts. Based on several pilot studies in which we evaluated the readability of specific design criteria, we developed a total of 21 tactile bar charts, line charts, scatterplots and pie charts, which we evaluated with 48 blind and visually-impaired people. In [?] we presented first results of this remote study. In this paper, we describe our study and results in detail for specific design parameters that have not been discussed in detail in prior research. In addition, we investigate whether characteristics of the user group influence the reading performance. 


\section{Remote User Study with Blind and Visually Impaired People}

For a remote user study we mailed the tactile charts to participants. Accordingly, they were free to perform the study at any time and place. This design avoids traveling to far away or to unknown places. Participants could work in their personal setup and use their own assistive technologies. We reached a large number of diverse participants who completed the study based on our instructions.

\subsection{Study Design}

Within the remote study, participants should complete an online survey and simultaneously explore the tactile charts. We emailed invitation to blind and visually-impaired people in Germany and posted invitations in social network groups, forums and mailing lists. We also asked associations of blind people as well as schools for the blind for participation. In addition, we attached the invitation to the study as black print and as a Braille version. Interested people could contact us to receive the materials by mail. In addition, they obtained a detailed description about the study via e-mail. Each participant received the following materials:

- A brief description about the study in Braille

- A description how to mail the materials back to us (including an address label with our address on it)

- 21 individually-sorted tactile charts

- Personalised URL to the online survey with an access key

We put Braille labels on the envelope so that blind participants could identify the envelope independently. We requested the participants to send the materials back to us after performing the study to save paper resources and re-use them. It was advised to perform the study within at most a few days without longer breaks. We instructed the participants to carry out the study without any aids (e.g. ruler, other people).

Overall, our mailing consists of seven bar charts, six line charts and three pie charts as well as five scatterplots. The charts were randomised by chart type (the paper on the top was the first). Each chart was identifiable by a number within a box in the upper left corner and referenced within the online survey. Accordingly, participants could always check ordering of the charts. We defined six randomisation configurations to avoid learning effects while exploring. Only pie charts were not randomised; rather, they were always last in the pile. The structure and elements of pie charts strongly differ from other chart types. Minor learning effects were expected. In addition, pie charts are the least suitable for data analysis because they can only represent a small amount of data. All six permutations of line charts, bar charts and scatterplots were used. The order of charts within the pages for each chart type was fixed. The full completion of two out of four chart types (where pie charts are always in fourth position) 


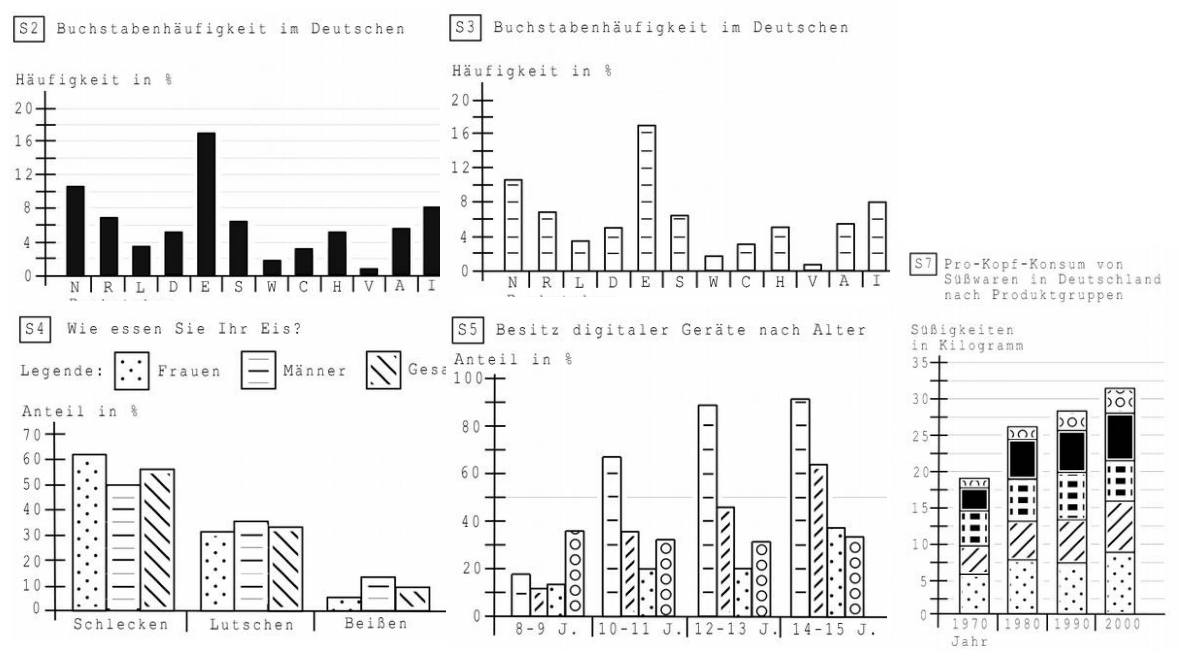

Fig. 1. Simple, grouped and stacked bar charts that we generated for evaluation (from left to right: S2, S3, S4, S5, S7)

were mandatory to receive a voucher amounting to 15 Euros for participation. Finally, the participants were asked to complete an additional survey to rate the study design and its content.

\section{$3.2 \quad$ Stimuli}

The charts represent real data sets to stimulate interest in the results. Charts were embossed on A4 format, mostly in landscape orientation (except from the stacked bar chart). While some charts include a legend on the same sheet, three charts come with a legend on a seperate sheet. Each chart has a title at the top, a box with an ID as well as labelled axes. The sizes and design of chart elements and distances are based on existing guidelines [?,?,?], the results of our own analysis (e.g. [?]) and pilot studies. Repeating chart elements were designed consistently. Axes and tick marks are about $1 \mathrm{~mm}$ (one embossed line). The line styles and textures used are well evaluated by Prescher et al. [?,?] for general use. Moreover, we evaluated various design parameters that have not yet been considered. Therefore, we developed charts with different design characteristics to evaluate their effectiveness. In addition, we compared design alternatives directly where we developed two charts that only differ in one design parameter.

Bar Charts. We generated three simple bar charts (S1-S3), three grouped bar charts with multiple data sets (S4-S6) and the stacked bar chart S7 (see Figure ??). The wider bars have a width of about $2 \mathrm{~cm}$, the smaller are $1 \mathrm{~cm}$ wide [?]. While bars of S2 are filled, S3 makes use of a texture consisting of horizontal 

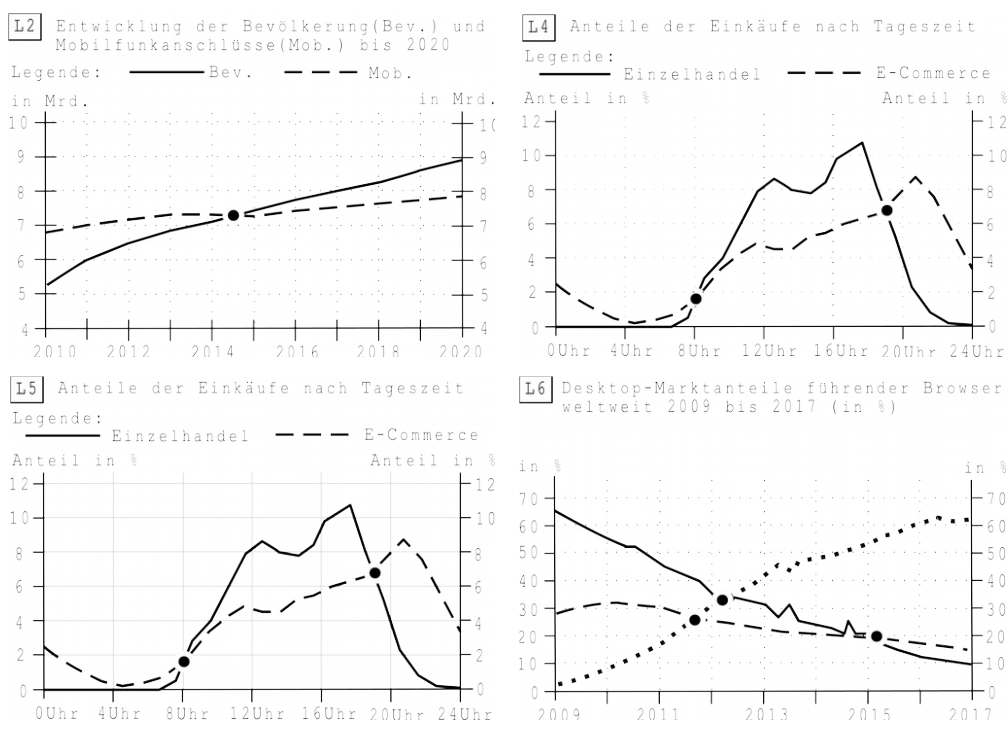

Fig. 2. Line charts used for evaluation (from left to right: L2, L4, L5, L6)

grid lines to mark scale units. S2 has thin grid lines in the background of the chart area. The charts represent the frequencies of usage of letters in German. Condition S4 is a grouped bar chart with three textures. The bar width is $2 \mathrm{~cm}$ in accordance with accepted guidelines. S5 represents the same textures whereby one texture was added. The bars of S5 are only half as wide as those in S4. In contrast to S5, S6 increased distances between all bars within each group. The stacked bar chart S7 was embossed in portrait format and utilizes five textures in every sub-segment. The chart contains segments in a suitable height as well as very small segments. The chart represents the per capita consumption of sweets in different years.

Line Charts. We created six line charts, each of which represents between one to three data lines (see Figure ??). The vertical axis is duplicated in all charts. We used solid, dashed and dotted line styles. L2, L3, L4 and L6 have a thin, dotted grid. Two pairs were generated. With L2 (marked) and L3 (not marked), we compared the effectiveness of marking intersection points with point symbols. While L4 makes use of thin, dotted grid lines, L5 uses solid ones. Both charts represent one solid and one dashed data curve with two intersection points. S6 is the most complex line chart, containing three intersection points of three different lines.

Scatterplots. Four tactile scatterplots (P1, P2, P3, P5) with one to three data sets were developed (see Figure ??). P2 and P3 differ in the use of grid lines. Because grid lines often cause confusion in scatterplots, we used an incised grid 

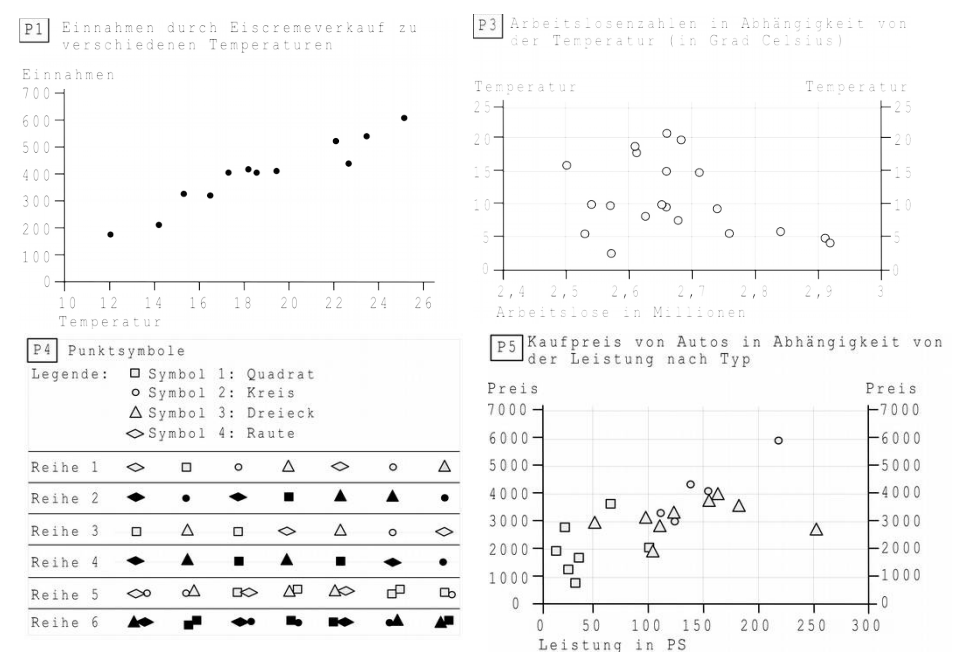

Fig. 3. We generate in fact four scatterplots and one condition to evaluate point symbols separately (from left to right: P1, P3, P4, P5)

in condition P3. Therefore, the grid lines were embossed separately on the background of the sheet. Data points were embossed with a high outline and a filling with a much lower high. Points have a diameter of about $0.6 \mathrm{~cm}$. Additionally, we evaluated the readability of four alternative point symbols (square, rhombus, triangle, circle). Therefore, we randomly arranged seven symbols in each of six rows. Each row contains all four symbols. Two design alternatives were used: first, the symbols have a high outline and were filled with much lower filling (row 1,3,5); and second, the symbols and outline were embossed with equal high (row 2,4,6). The first two rows compare the readability of single symbols in both design conditions. The third and fourth row added a thin grid line to the symbols to evaluate their influence on symbol readability. The final two rows represent two overlapping symbols at a time. P5 represents three data sets with the use of circles, squares and triangles. The symbols are not described, so participants should interpret their meaning by themselves. Especially in the centre of the chart, several symbols overlap each other. In order to ensure readability, we add an incised grid embossed on the background.

Pie Charts. As pie charts require many textures, we developed just three pie charts. K2 is divided into four slices and uses the same number of textures. Textures are separated by white spaces. K3 omits textures and is divided into seven outlined segments. Labels were placed within the slices. If required, labels were positioned outside the chart. On the outer side of the circle, we added markers in $5 \%$ steps. 


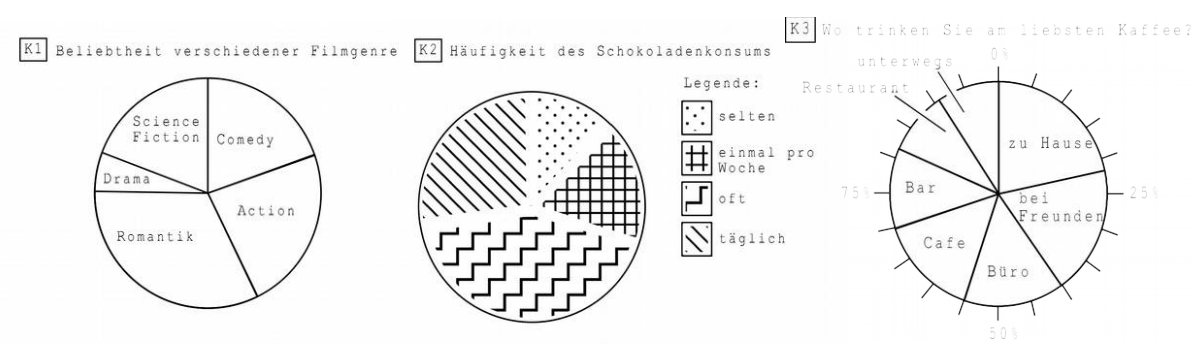

Fig. 4. Three pie charts - with and without textures - used in the study (from left to right: $\mathrm{K} 1$ to $\mathrm{K} 3$ )

\subsection{Procedure of the Online Survey}

We carried out a pre-test to evaluate the accessibility and feasibility of the study with one blind participant. Therefore, the participant checked the printed Braille texts. Afterwards, the participant performed the online study while exploring the charts. The participant used own setup, in particular a laptop with a standard keyboard and a refreshable Braille display as well as JAWS. The study investigator could monitor on a display the progress. The pre-test lasted about 6 hours, during which issues were discussed immediately. Several issues were identified and fixed later.

At the very beginning of the survey, participants were asked about their experiences with tactile charts. They were instructed to explore the materials independently. The exploration process starts with a training phase that can be skipped when participants are familiar with tactile charts. We provided a training chart with a detailed description containing the general structure and content of the chart. Participants should explore one chart after another and control the ID placed within the box in the upper left corner of each chart. Specifications for each chart type were introduced by a training chart (S1, L1, P1, K1). Participants received a description of the chart if they did not understand it independently. Accordingly, we ensure that every participant could understand the chart type in general.

For most of the charts, we used the following six question types:

1. QReadability: Judgement of the understanding of the chart (without instructions): Agreement with the statement "I could understand the content and the structure of the chart very well." on a five-point Likert scale from "Fully agree" to "Not agree". Those who did (rather) not agree with the statement received an additional description of the chart.

2. Q $\mathrm{Q}_{\text {Nominal }}$ : Questions concerning the retrieval of nominal values such as naming categories for minimum and maximum values or comparisons (1 to 4 per chart).

3. Q Accuracy: Questions on metric values such as reading out values, comparing different data sets or single values, finding extremes or intersection points (1 to 4 per chart). 
4. Q Qesign: Statements concerning the readability and design of specific design parameters that should be rated on a five-point Likert scale.

5. Q Q Rate: Rating of the chart on a scale from 1 to 6 .

6. $\mathrm{Q}_{\text {Text }}$ : Free-text input field for additional comments.

The participants were allowed to finish the study already after two of four chart types. Finally, demographic data were recorded, in particular age, level of blindness, profession and Braille reading skills.

\subsection{Participants}

Overall, 48 participants (43 blind, 5 visually-impaired) completed our study. $50 \%$ of the participants were born with a visual impairment, $15 \%$ became blind at an age less than 10 years, while $4 \%$ are late-blinded (becoming blind at an age between 41 and 60). The participants are heterogeneous concerning different properties. The age of the participants ranged from 16 to 77 years with a median of 44. Overall, 27 females and 21 males completed the study. Only one participant had no experience with reading Braille. About $70 \%$ indicated having good Braille reading skills. The professions of participants are diverse, with teachers, students, pupils as well as three pensioners taking part. Besides professions in the information technology field (e.g. computer science, software developer), there were also participants from the social domains as well as Braille transcribers.

Overall, $74 \%$ have experience in exploring tactile charts. $47 \%$ of them stated having "very high" or "high" experience. Of the twelve participants who had no experience with tactile charts, eight knew the general structure of charts, while four participants did not know anything about charts. Above all, most participants had experience with exploring tactile bar charts $(89 \%)$ and tactile line charts $(94 \%)$. Furthermore, $71 \%$ had experience with tactile pie charts. Scatterplots $(23 \%)$ and stacked bar charts $(31 \%)$ were still unknown to most participants. By comparison, among participants without experience with exploring tactile charts, line charts were only known by $67 \%$. Participants with tactile chart experience (35 of 48) mostly used charts to gain access to a visual chart $(63 \%)$ and read out values $(69 \%)$. More than half of them use tactile charts to gain an overview, while only $31 \%$ made use of tactile charts to analys e data. In addition, swellpaper and Braille printers are most familiar for participants (71\%). $57 \%$ explore tactile graphics primarily made from thermoformed foils, while $29 \%$ primarily use a graphic embosser as used in our study.

\subsection{Statistical Measurements}

First, we identified unclear answers, in particular when ranges or multiple values were entered instead of single values or spelling mistakes. They were excluded from the calculations. For each chart, we computed frequencies for QReadability, the relative number of Correct Answers for $\mathrm{Q}_{\text {Nominal }}$ as well as the Mean Error

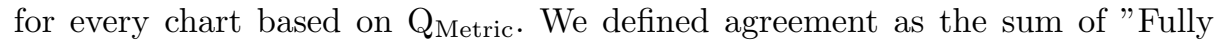
agree" and "Rather agree" for $Q_{\text {Readability }}$ and $Q_{\text {Design }}$. We computed the Mean 
Error as the average of deviations from all answers to the correct value based on valid numerical values. For the analysis, we excluded incorrectly answered responses as Mean Error corrected as follows. For example, when the value of the highest value instead of the smallest value (mentioned in the question) was named, the question was misunderstood. We also excluded all answers outside the value range of the axis. In some cases, these answers could be identified by the corresponding $\mathrm{Q}_{\text {Nominal }}$. For example, participants were asked to find two bars with the same height. Afterwards, participants should name the value of the two bars with the same height. If the first question was answered incorrectly, the required value for second question would be incorrect. Q Design measures subjective data and addresses chart-specific design parameters. However, some questions are identical for different charts so that e.g. the readability of bars can be compared among charts with different design aspects. Finally, we calculated the median for ratings. In addition, we compared the results by dividing participants into groups concerning the degree of experience with exploring tactile charts $\left(\mathrm{E}_{1}\right.$ : have experiences, $\mathrm{E}_{2}$ : no experiences) and the age of blindness $\left(\mathrm{B}_{1}\right.$ : birth-blind, $\mathrm{B}_{2}$ : not birth-blind). We used the Mann-Whitney $\mathrm{U}$ test for pairwise significant tests and non-parametric Friedman test for multiple comparisons.

\section{Results}

Overall, 37 participants completed all chart types, among whom 11 participants performed two chart types completely. Participants needed a minimum of 2 hours to complete the whole study. We estimate based on the measured times and comments that most participants needed 3 to 4 hours. In this section, we first summarise results of all charts, before proceeding in detail and showing the results for specific conditions per chart type.

\subsection{Overall Results}

First, we analysed how well participants understood the chart contents individually (QReadability). Overall, about $59 \%$ fully agree with this statement. $23 \%$ of participants "Rather agree", while on average $1.5 \%$ "Fully disagree" with the statement. Concluding from this, most of the charts are well understandable without the need for additional instructions. $\mathrm{K} 1$ has the highest rate with agreement of $100 \%$ (sum of "Fully agree" and "Rather agree"), followed by L2 with $90 \%$. Overall, less complex charts (e.g. training chart, first three charts of each chart type) have a higher agreement rate $(>85 \%)$ than more complex charts (e.g. line charts with three data lines, stacked bar charts). A non-parametric Friedman test of differences in agreement among $\mathrm{E}_{1 / 2}$ and $\mathrm{B}_{1 / 2}$ was not significant $(\mathrm{p}=.9)$. Furthermore, we compared the mean rate of Correct Answers for $\mathrm{Q}_{\text {Nominal }}$ among all charts. On average, $80 \%$ of nominal questions were answered correctly. The differences between groups $\mathrm{B}_{1 / 2}$ and $\mathrm{E}_{1 / 2}$ are not significant $(\mathrm{p}=.4)$. L2 $(100 \%), \mathrm{K} 3(95 \%)$ and S5 $(95 \%)$ achieved the best correct rate, where only one nominal question was asked. The scatterplot P2 has the 
lowest rate with $49 \%$. Here, participants had problems with counting data points within a specific range without having a grid.

Overall, there is a significant difference $(\mathrm{p}=.008)$ between the Mean Error

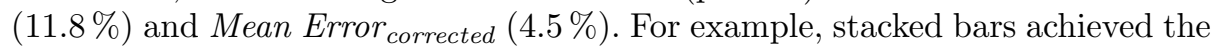
worst results with a Mean Error of $22.7 \%$ (see Table ??) while the corrected error is much smaller (5.4\%). This can be explained by the fact that many participants indicated the value of the whole column if the value for a sub-segment was asked

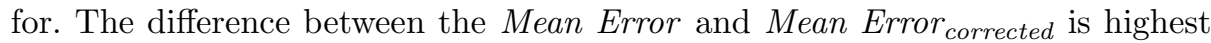
among birth-blind participants (about 11\%). There was neither a significant

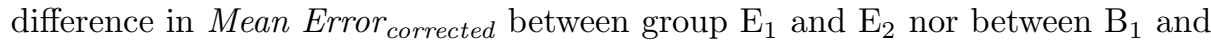

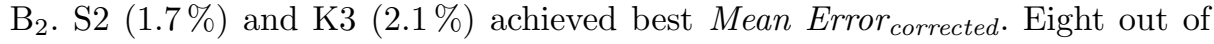
fourteen charts have a corrected error rate below $5 \%$ which indicates a high level of correctness when reading out values. Scatterplots achieved the highest corrected error rate of $6 \%$, followed by line charts with $5.8 \%$. These findings could not be generalised for all chart types because we cannot quantify the level of difficulty for each question of every chart.

However, finally we compared the ratings that participants assigned to every chart. This may reflect individual preferences as well as difficulties created by a chart. Overall, the median of all ratings is 2 ("Good"). The pie chart K3 rated with the best mark of 1. L4, L6 and K2 are rated worst by a mark of 3 as median. All results depend on several parameters, such as the number of questions, level of difficulty and number of charts of this chart type. We present detailed results for specific charts in the following.

\subsection{Bar Charts}

Bar charts achieved a Mean Error corrected $_{\text {of }} 3.2 \%$. We investigated the design of fillings and grid lines, distances between bars, sizes of bars and textures.

Design of grid lines (S2, S3). S3 makes use of a horizontal texturing to replace grid lines, while S2 has filled bars and conventional grid lines. S2 leads to more Correct Answers as well as a lower Mean Error rate (see Table ??). Because grid lines within the bars are unfamiliar to most participants, fewer participants recognised them in S3 than in S2, which may be the reason for the higher error rate. Recognising bars is more suitable in condition S2 (100\%) than in condition S3 (89\%). Moreover, participants rate reading out values and

Table 1. Direct comparison of Correct Answers (in \%), Mean Error rate (in \%), corrected Mean Error rate (in \%) and ratings of all charts.

\begin{tabular}{|c|c|c|c|c|c|c|c|c|c|c|c|c|c|c|}
\hline Chart ID & S2 & S3 & $\mathrm{S} 4$ & S5 & S7 & $\mathbf{P 2}$ & P3 & P5 & L2 & L4 & L5 & L6 & K2 & K3 \\
\hline Correct Answers & 74 & 77 & 71 & 95 & 74 & 49 & 76 & 94 & 100 & 81 & 81 & 82 & 77 & 95 \\
\hline Mean Error & 2.4 & 3.2 & 3.1 & 4.2 & $\overline{22.7}$ & 13.5 & 12.6 & 60.8 & 17.7 & 4.4 & 5.7 & 7.3 & 4.8 & 3.1 \\
\hline Mean Error & 1.7 & 3.0 & 2.2 & 3.9 & 5.4 & 7.0 & 6.3 & 4.8 & 7.7 & 4.4 & 5.7 & 5.2 & 4.8 & 2.1 \\
\hline Rating (Median) & 2 & 2 & 2 & 2 & 2.5 & 2 & 2 & 2 & 2 & 3 & 2 & 3 & 3 & 1 \\
\hline
\end{tabular}


comparing data among bars as much higher in S2. Overall, only $15 \%$ of the participants prefer the design in condition S3. We conclude that condition S2 is more effective than condition S3, although thin grid lines in bar charts are suitable to read out values. Other supporting users elements should be explained in advance. One participant recommended combining both types of grid lines.

Comparison of bar width (S4, S5). In this comparison, we investigated the influence of the column width on the readability of textures. Participants could recognise bars and textures better in S4 than in S5 (see Table ??). The corrected Mean Error is lower in S4 (no significance). Width of bars influences the readability of the chart. Textures and bars in condition $\mathrm{S} 4$ are better readable.

Table 2. Comparison of Agreement (sum of "Fully agree" and "Rather Agree") for design statements of condition $\mathrm{S} 4$ and $\mathrm{S} 5(\mathrm{~N}=32)$.

\begin{tabular}{|l|l|l|}
\hline Statement & S4 & S5 \\
\hline I could recognize all bars immediately. & 93.5 & 77.3 \\
\hline I could distinguish all textures easily. & 95.7 & 77.8 \\
\hline I could reference all textures in legend. & 95.7 & 75.5 \\
\hline Help lines inside the bars were helpful to read out values. & 73.91 & 77.8 \\
\hline
\end{tabular}

Distance between bars (S5, S6). We investigated the influence of distances between bars on the readability of bars. Adding distances between bars in groups increases the comparability of values between bars as well as the readability of single bars for most participants (see Table ??). By contrast, about one-quarter of participants prefer omitting distances.

Table 3. Comparison of Agreement (sum of "Fully agree" and "Rather Agree") and Disagreement (sum of "Fully disagree" and "Rather Disagree") of condition S5 and S6 in $\%(\mathrm{~N}=31)$.

\begin{tabular}{|l|l|l|}
\hline Statement & Agree & Disagree \\
\hline Single bars are better distinguishable in S6 than in S5 & 71.0 & 24.5 \\
\hline The comparison among bars is better in S5. & 27.0 & 60.0 \\
\hline No difference in readability between S5 and S6. & 34.0 & 52.3 \\
\hline I prefer condition S5. & 22.7 & 68.2 \\
\hline
\end{tabular}

Stacked bar charts (S7). Most participants were unfamiliar with stacked bars $(69 \%)$. More than $41 \%$ fully or rather agree that it was difficult to distinguish all textures within the bars. Textures in stacked bar charts reduce the readability 
(61\%). By contrast, only $22 \%$ prefer omitting textures (simply using the order of segments in a bar for orientation). Help lines are suitable to read out values in stacked bar charts (83\%). When participants were asked to read the value of the upper section of a bar, $41 \%$ indicated the total value of the bar. The meaning of stacked bar charts can be misunderstood. Participants reported high mental effort when analysing data in stacked bar charts.

\subsection{Line Charts}

We investigated the design of intersection points and grid lines in line charts as well as the readability of a complex line chart with three data curves.

Design of intersection points (L2, L3). Participants rated both designs roughly equally. In L2, $80 \%$ could recognise the intersection point very easily. $14 \%$ cannot find any difference in the readability of intersection points. By contrast, $56 \%$ prefer the marked intersection point in L2, whereas $28 \%$ also prefer condition L3. Therefore, marking single intersection points may influence chart readability less. There is little evidence that marking intersection points increases the readability of crossing data lines.

Design of grid lines (L4, L5). Participants rate solid grid lines in L5 better than dotted grid lines in L4 (see Table ??). Solid grid lines are significant better recognised $(\mathrm{p}=.03)$ than dotted grid lines (see Table ??). The design of the grid lines also influences the perceptibility and traceability of the data lines. Overall, $86 \%$ of the participants prefer solid grid lines in line charts, especially when dashed and solid data lines were used.

Table 4. Comparison of Agreement (sum of "Fully agree" and "Rather Agree") for design statements of condition L4 and L5 in \% ( $\mathrm{N}=42)$.

\begin{tabular}{|l|l|l|}
\hline Statement & L4 & L5 \\
\hline I could recognize all lines easily. & 69.1 & 78.5 \\
\hline It was easy to distinguish lines. & 76.7 & 79.5 \\
\hline I could follow lines easily. & 72.1 & 85.7 \\
\hline I could recognize help lines very easy. & 69.8 & 90.5 \\
\hline Help lines were very helpful to read out values. & 80.9 & 92.6 \\
\hline I could recognize all intersection points very well. & 74.8 & 83.3 \\
\hline
\end{tabular}

Complex line charts (L6). L6 represents three data lines (solid, dotted, dashed) as well as three intersection points. It is very difficult to read this chart because solid and dashed line are very close to each other. Furthermore, a thin, dotted grid was added. Lines in this chart can be recognised very well with a rate 
of $56 \%$. Line tracing is easiest for the solid line ( $83 \%$ of agreement), while the dashed $(57 \%)$ and dotted lines $(60 \%)$ are rated as roughly equal. In addition, $31 \%$ agree that it was difficult to distinguish between dotted and dashed lines. For $82 \%$, it was easy to distinguish data curves and grid lines. Grid lines are used by $88 \%$ to read out values. Only $63 \%$ recognise intersection points very well.

\subsection{Scatterplots}

We compared the usage of grid lines in scatterplots as well as point symbols and the readability of complex scatterplots.

Usage of grid lines (P2, P3). In the first condition P2 (without grid lines), participants gave $20 \%$ fewer Correct Answers than in the second condition (with grid lines). Especially counting data points in a certain area is challenging without grid lines (about 30\% fewer Correct Answers). It was easy to recognise single data points for $90 \%$ of participants. Help lines in P3 do not influence the readability of data points $(97.3 \%$ ), while they could be recognised very well by $93.1 \%$. In condition P2, it was difficult for $37 \%$ to read out precise values, while in condition P3 only $27 \%$ agree with it. From these findings, it can be concluded that grid lines clearly influence the readability of scatterplots. Incised grid lines are suitable to support reading out values, whereby they can be recognised very well and do not influence the readability of data points provided that they have a suitable size.

Point symbols (P4). P4 compares four point symbols (square, circle, rhombus, triangle), whereby the task was to name the correct symbols within each row. In the last two rows, participants should name one of the two symbols that overlap each other. Overall, the first four rows have similar recognition rates. In addition, $48 \%$ preferred the outlined symbols over filled points. In the first row, participants named $90 \%$ of symbols correctly, which is the highest value over all rows. Overlapping symbols in rows five and six leads to a lower correct rate of answers $(71 \%)$. Here, overlapping reduced the correct recognition of tactile symbols by about $20 \%$. The overall correct answers per symbol type are similar. In general, $75 \%$ of participants could distinguish all symbols very well. However, $32 \%$ state that the identification of symbols in rows five and six was very difficult. For $46 \%$ of participants, it was difficult to distinguish between the rhombus and square. In additional comments, several participants reported challenges in separating square and circle symbols. One participant proposed combining both design alternatives in one chart when multiple data sets are shown.

Complex scatterplots (P5). Overall, this chart has the lowest Mean Error ${ }_{\text {corrected }}$ and Correct Answers for of all scatterplots (see Table ??). The high uncorrected 
Mean Error can be explained by a large outlier, which probably results from a typo by one user. P5 has a mean corrected error rate of $4.8 \%$. Only one quantitative question was taken into account. $36 \%$ could recognise overlapping symbols easily, while $82 \%$ could identify the number of overlapping symbols correctly. $75 \%$ of participants agreed that triangles and circles could be easily distinguished. Almost one-third had problems recognising the distribution of the data points. Nearly all participants used incised help lines and rated them as very useful. Additionally, grid lines did not interrupt the recognition of symbols $(72.7 \%)$.

\subsection{Fillings in Pie Charts (K2, K3)}

With the pie charts, we compared the design of segments as well as the use of tick marks. K3 is the best-rated chart overall (median 1), while K2 is one of the worst-rated charts (median 3). K3 leads to many more Correct Answers and fewer errors. $78 \%$ could distinguish all textures in K2 well. Accordingly, only $24 \%$ agree that textures in slices increase estimating their sizes. There is also a strong difference relating the readability of slices, whereby $89 \%$ could recognise all slices in K3, compared with $59 \%$ in K2. Additionally, $17 \%$ prefer textures in pie charts over outlined slices. Despite the fact that K3 makes use of different label types, $84 \%$ of participants could assign labels to slices very well. Furthermore, tick marks on the outer circle of the chart are useful $(87 \%)$. Overall, textures in pie charts strongly influence the readability of the chart and thus they should be omitted in pie charts. In addition, they are not suitable to represent more than five single values, in contrast to outlined slices.

\subsection{Limitations}

The results reflect the effectiveness of tactile grouped and stacked bar charts, line charts, scatterplots as well as pie charts. Due to the remote study design, we do not know the reasons for any incorrect answers. The results may be biased by misunderstandings of the question, typing errors or handling problems of the online questionnaire. Accordingly, we conducted a manual error analysis for Mean Error calculation and identified answers with correct value. Especially typing errors (e.g. comma errors) lead to strong outliers, which strongly influenced the final results. Answers outside the value range of axes were excluded. On the other hand, we cannot guarantee that the participants did carry out the study on their own, even if they were instructed to do so. Nominal questions can be measured well with this study design because they provide evidence of the understanding and readability of the chart without instruction. Reasons for incorrect answers can at least be provided by text comments. Moreover, we could not measure the time that it took the participants to answer questions. Therefore, efficiency cannot be measured with this study. Nevertheless, despite $26 \%$ of participants having never explored a tactile chart before, even complex charts could be read and understood by most participants. 
In order to ensure the feasibility of the study, we provided many explanations and - if needed - chart descriptions. This replaces the study leader's additional explanation in laboratory studies. In order to obtain information about user experiences, we invited the participants to rate the study design in a separated short questionnaire.

\section{Evaluation of the Study Design}

We identified thirteen aspects that are important in remote studies with blind people. All participants (28) rate the whole study design, the chart descriptions as well the communication as "very good" or "good". While the personal benefit from the study is rated at $77 \%, 92 \%$ rate the fun factor with mark 1 or 2 . Information about the study, materials, accessibility and comprehension of the questions were rated very high with more than $95 \%$. By contrast, the duration to perform the study achieved the worst rating with $65 \%$. Overall, we receive very positive feedback. Some participants reported that they had learned much while performing the study, while others commented on the preparation of materials, which gave them a safety feeling. One participant state that he/she learned within the study how tactile charts could provide a benefit. Another person expressed the wish to produce such kinds of charts oneself. Especially those participants who had no prior experience with tactile charts rate the tutorial at the beginning of the study as very helpful. Twenty-one out of 28 participants read it completely. Sixteen participants learned the structure or rather the exploration of charts with the help of the training phase (including tutorial and charts). Many participants also commented on the long duration of the study and the high level of effort that performance required. Several participants recommended not to use computer Braille in tactile charts. One participant stated it would be better to omit the number sign instead of using computer Braille when a space is required. In addition, numbering questions is very helpful.

\section{Discussion and Outlook}

We have analysed quantitative and subjective data from 48 blind and visuallyimpaired participants about specific design properties as well as the readability of tactile charts. We ascertained that there are individual user preferences that the design should fit. In the following, we will summarise the major findings:

- Solid grid lines are suitable in bar charts. Grid lines within bars can be used but should be explained in advanced.

- Distances between bars of a group can increase the comparability of values between bars and the recognition of single bars.

- Wider bars with texture are preferred over thinner ones with texture. Even thin bars (about $1 \mathrm{~cm}$ ) with textures are readable.

- It is advised to use as few textures as possible in stacked bar charts. Stacked bar charts should only be used if the value of the sum of a bar is very interesting; otherwise, the use of grouped bars is recommended. 
- Marking intersection points with a circle in line charts does not strongly influence the readability of the chart.

- The usage of solid help lines instead of dotted grid lines is recommended in line charts.

- Solid line style for curves are best readable in line charts, even if solid help lines were used.

- Duplicated vertical axes increase reading out values in line charts and scatterplots.

- Help lines in scatterplots are recommended for reading out values and counting points.

- Incised help lines are suitable in scatterplots with data points of an appropriate size (about $1 \mathrm{~cm}$ ).

- Three to four different symbols in scatterplots are readable. Circles and triangle are very well distinguishable. Rectangles and rhombus shapes and more specifically circles and squares should significantly differ.

- Omitting textures in pie charts increases the readability.

- Adding tick marks to pie charts increases the reading accuracy.

We have shown that the design of tactile charts (e.g. grid lines, design of elements) influences the readability, depending on the whole design, the chart type and the represented data. Blind people are able to read trends, outliers, clusters and specific values out of charts. Even complex charts with three data lines or multiple symbols are readable for most participants, including novices in tactile chart reading. We found no significant difference in performance (mean error, correct answers) of birth-blind and not-birth-blind participants. Surprisingly, we could not demonstrate a significant difference between experienced and inexperienced participants. To conclude, tactile charts are usable for commonly-known chart types, whereby blind people are able to analyse data effectively. While other authors have identified the effectiveness of tactile charts by comparing them with other data-representing methods (e.g. a tactile or digital table), we will compare the calculated effectiveness of the charts with another user group. Accordingly, we are currently conducting a comparative study in which sighted participants receive the same charts (black printed, not embossed) and questions as blind participants. The aim of this study is to compare the effectiveness among blind and sighted people when analysing charts. Additionally, the results of this study have highlighted many more design alternatives we will take into account when re-designing charts for further user studies. Furthermore, we will implement well-chosen guidelines for tactile charts based on these results.

Besides improving the production process to increase the availability of tactile charts, there is a need for more user studies in tactile chart design. Especially the design of more chart types with useful data and tactile variables - such as line width, symbols, line styles, and help elements - should be explored in detail. The results can be used to design tactile charts effectively for different use cases that support blind people by fulfilling many tasks that are needed in several professions. 


\section{References}

1. Aldrich, F.K., Parkin, A.J.: Tangible Line Graphs: An Experimental Investigation of Three Formats Using Capsule Paper. Hum. Factors 29(3), 301-309 (Jun 1987). https://doi.org/10.1177/001872088702900304, https://doi.org/10.1177/001872088702900304

2. Barth, J.L.: Incised grids: Enhancing the readability of tangible graphs for the blind. Human factors 26(1), 61-70 (1984)

3. Braille Authority of North America, Canadian Braille Authority: Guidelines and Standards for Tactile Graphics (2011)

4. Cleveland, W.S.: Visualizing Data. Hobart Press (1993)

5. Culbert, S.S., Stellwagen, W.T.: Tactual Discrimination of Textures. Perceptual and Motor Skills 16(1957), 545-552 (1963), http://www.ncbi.nlm.nih.gov/pubmed/14024190

6. Engel, C., Weber, G.: Analysis of tactile chart design. In: Proceedings of the 10th International Conference on PErvasive Technologies Related to Assistive Environments. pp. 197-200. PETRA '17, ACM, New York, NY, USA (2017). https://doi.org/10.1145/3056540.3064955, http://doi.acm.org/10.1145/3056540.3064955

7. Engel, C., Weber, G.: Improve the accessibility of tactile charts. In: IFIP Conference on Human-Computer Interaction. pp. 187-195. Springer (2017)

8. Engel, C., Weber, G.: A user study to evaluate tactile charts with blind and visually impaired people. In: International Conference on Computers Helping People with Special Needs. pp. 177-184. Springer (2018)

9. Goncu, C., Marriott, K.: Tactile chart generation tool. Proceedings of the 10th international ACM SIGACCESS conference on Computers and accessibility - Assets '08 p. 255 (2008). https://doi.org/10.1145/1414471.1414525

10. Goncu, C., Marriott, K., Aldrich, F.: Tactile diagrams: Worth ten thousand words? In: Goel, A.K., Jamnik, M., Narayanan, N.H. (eds.) Diagrammatic Representation and Inference. pp. 257-263. Springer Berlin Heidelberg, Berlin, Heidelberg (2010)

11. Goncu, C., Marriott, K., Hurst, J.: Usability of accessible bar charts. In: Diagrammatic Representation and Inference, pp. 167-181. Springer (2010)

12. Lederman, S.J., Campbell, J.I.: Tangible graphs for the blind. Human Factors: The Journal of the Human Factors and Ergonomics Society 24(1), 85-100 (1982)

13. Nolan, C.Y., Morris, J.E.: Improvement of Tactual Symbols for Blind Children. Final Report. p. 88 (1971), http://eric.ed.gov/?id=ED070228

14. Paneels, S., Roberts, J.C.: Review of designs for haptic data visualization. IEEE Transactions on Haptics 3(2), 119-137 (2010). https://doi.org/10.1109/TOH.2009.44

15. Prescher, D.: Guidelines for Image Descriptions and Tactile Graphics (2016), http://nbn-resolving.de/urn:nbn:de:bsz:14-qucosa-196167

16. Prescher, D., Bornschein, J., Weber, G.: Consistency of a tactile pattern set. ACM Trans. Access. Comput. 10(2), 7:1-7:29 (Apr 2017). https://doi.org/10.1145/3053723

17. Round Table on Information Access for People with Print Disabilities Inc.: Guidelines on Conveying Visual Information (2005), http://printdisability.org/guidelines/guidelines-on-conveying-visual-information$2005 /$

18. Schuffelen, Marco: On editing graphics for the blind (2002), http://piaftactile.com/docs/Tactile_Graphics_Manual.pdf 
19. Shneiderman, B.: The eyes have it: a task by data type taxonomy for information visualizations. In: Proceedings 1996 IEEE Symposium on Visual Languages. pp. 336-343 (Sep 1996). https://doi.org/10.1109/VL.1996.545307

20. Watanabe, T., Mizukami, H.: Effectiveness of tactile scatter plots: Comparison of non-visual data representations. In: Miesenberger, K., Kouroupetroglou, G. (eds.) Computers Helping People with Special Needs. pp. 628-635. Springer International Publishing, Cham (2018)

21. Watanabe, T., Yamaguchi, T., Nakagawa, M.: Development of software for automatic creation of embossed graphs: Comparison of non-visual data presentation methods and development up-to-date. Lecture Notes in Computer Science (including subseries Lecture Notes in Artificial Intelligence and Lecture Notes in Bioinformatics) 7382 LNCS(PART 1), 174-181 (2012). https://doi.org/10.1007978-3-642-31522-0_25 